\title{
Design of Detection-Jamming Shared Waveform Based on Virtual Force Field Algorithm
}

\author{
GuoMiao Xiong ${ }^{1}$, Yunpeng $\mathrm{Li}^{1}$, and Chao Chen ${ }^{1}$ \\ ${ }^{1}$ Chinese People's Liberation Army
}

July 27, 2021

\begin{abstract}
Due to the technical barriers between radars and jammers and the poor performance of the traditional detection-jamming shared signal in integrated radar-electronic warfare systems, a new detection-jamming shared signal waveform based on the virtual force field algorithm (VFFA) is proposed in this paper. First, a multi-objective and multi-dimensional characteristic parameter optimization model, based on a virtual force field, is established, and then the design principle of the shared signal is presented in detail. The simulation results show that the detection-jamming shared signal based on the VFFA presents the deceptive jamming of multiple false targets in non-collaborative radar. Further, there is better detection performance with the advantages of multiple pulse repetition frequency (PRF) and pulse accumulation number, which are highly sensitive to the multi-jagged PRF signals emitted by the non-collaborative radar. According to the VFFA described in this paper, the optimum detection-jamming shared signal waveform can be output in real time for specific free space targets, to improve the efficiency of integrated radar and electronic warfare systems.
\end{abstract}

\section{Hosted file}

manuscript.pdf available at https://authorea.com/users/427758/articles/531880-design-ofdetection-jamming-shared-waveform-based-on-virtual-force-field-algorithm

\section{Hosted file}

figure1.docx available at https://authorea.com/users/427758/articles/531880-design-ofdetection-jamming-shared-waveform-based-on-virtual-force-field-algorithm

\section{Hosted file}

figure2.docx available at https://authorea.com/users/427758/articles/531880-design-ofdetection-jamming-shared-waveform-based-on-virtual-force-field-algorithm

\section{Hosted file}

figure3.docx available at https://authorea.com/users/427758/articles/531880-design-ofdetection-jamming-shared-waveform-based-on-virtual-force-field-algorithm

\section{Hosted file}

figure4.docx available at https://authorea.com/users/427758/articles/531880-design-ofdetection-jamming-shared-waveform-based-on-virtual-force-field-algorithm

\section{Hosted file}

figure5.docx available at https://authorea.com/users/427758/articles/531880-design-ofdetection-jamming-shared-waveform-based-on-virtual-force-field-algorithm 


\section{Hosted file}

figure6.docx available at https://authorea.com/users/427758/articles/531880-design-ofdetection-jamming-shared-waveform-based-on-virtual-force-field-algorithm

Hosted file

figure7.docx available at https://authorea.com/users/427758/articles/531880-design-ofdetection-jamming-shared-waveform-based-on-virtual-force-field-algorithm

\section{Hosted file}

figure8.docx available at https://authorea.com/users/427758/articles/531880-design-ofdetection-jamming-shared-waveform-based-on-virtual-force-field-algorithm

\section{Hosted file}

figure9.docx available at https://authorea.com/users/427758/articles/531880-design-ofdetection-jamming-shared-waveform-based-on-virtual-force-field-algorithm 